УДК 519.233.2

ISSN 1995-5499

DOI: https://doi.org/10.17308/sait.2021.3/3733

Поступила в редакцию 26.08.2021

Подписана в печать 20.11.2021

\title{
ОЦЕНКА ПАРАМЕТРОВ НЕЛИНЕЙНЫХ РЕКУРРЕНТНЫХ СООТНОШЕНИЙ
}

\author{
(c) 2021 Г. Ш. Цициашвили, М. А. Осипова ${ }^{\bowtie}$
}

Институт прикладной математики ДВО РАН

ул. Радио, 7, 690041 Владивосток, Российская Федерация

Дальневосточный федеральный университет

10, n. Аякс, о. Русский, 690922 Владивосток, Российская Федерация

\begin{abstract}
Аннотация. В настоящей работе построены оценки параметров нелинейных рекуррентных последовательностей по неточным наблюдениям. Речь идет о модели логистического роста, модели Рикера и дискретизированной модели Лоренца. В модели Лоренца дифференциальная задача была сведена к конечно-разностной схеме. Рассмотрены аддитивная и мультипликативная модели внесения ошибок в наблюдения, при этом распределения ошибок имеют не всегда нормальный закон распределения. Основная идея работы состоит в представлении параметров моделей через временные средние определенных функций и оценки этих средних по наблюдениям. Вопросы существования временных средних некоторых функций от модельных переменных являются предметом теории динамических систем. Они определяются наличием предельных циклов или предельных распределений динамической системы. Так как динамические системы наблюдаются на фоне случайных ошибок, то оцениваемые параметры выражаются через средние по траектории и через дисперсии ошибок наблюдений. Важным этапом в настоящей работе стало доказательство сходимости по вероятности оцениваемых параметров детерминированной системы к точным значениям. Эта процедура основана на классических вероятностных неравенствах типа неравенства Чебышева. Полученные результаты проверены в ходе вычислительных экспериментов, в которых строятся полигоны частот оцениваемых параметров и сравниваются с их точными значениями. Рассмотренные в работе модели динамических систем являются нелинейными, что затрудняет использование метода наименьших квадратов для оценки их параметров. Известные авторам результаты численного эксперимента по такой оценке параметров содержат достаточно большие ошибки. Тогда как предложенный в работе метод оценивания параметров нелинейных динамических систем является аналитически строгим.
\end{abstract}

Ключевые слова: нелинейные рекуррентные последовательности, оценки параметров, сходимость по вероятности, предельные циклы и распределения.

\section{ВВЕДЕНИЕ}

В настоящей работе построены алгоритмы оценивания параметров в нелинейных рекуррентных моделях динамических систем по неточным наблюдениям, что имеет важное теоретическое и практическое значение. Речь идет о модели логистического роста, модели

Осипова Марина Анатольевна e-mail: mao1975@list.ru
Рикера и дискретизированной модели Лоренца. Рассматриваемые модели привлекают к себе повышенное внимание со стороны биологов, физиков и метеорологов (см. обзоры литературы $[1,2])$. Они описывают динамику численности популяции, динамику изменения метеорологических параметров и т. д.

Основная идея работы состоит в представлении параметров моделей через временные средние определенных функций и оценки этих средних по наблюдениям. Это связано с тем, что различные процессы, описываемые Контент доступен под лицензией Creative Commons Attribution 4.0 License. The content is available under Creative Commons Attribution 4.0 License. 


\section{Г. Ш. Цициашвили, М. А. Осипова}

перечисленными моделями, наблюдаются на фоне случайных ошибок. Вопросы существования временных средних от модельных переменных являются предметом теории динамических систем [3-7]. Они определяются наличием предельных циклов или предельных распределений в динамической системе выделением притягивающего множества аттрактора, все траектории из некоторой окрестности которого стремятся к нему при (времени) стремящемся к бесконечности.

Временные средние для оценки параметров нелинейных рекуррентных последовательностей впервые были использованы в работе [8] применительно к модели Рикера с мультипликативной моделью внесения ошибок наблюдений и в работе [9]. Однако в этих работах не исследовалась аналитически сходимость построенных оценок к их точным значениям. Авторам не удалось найти информацию об оценке параметров через средние по траектории и об их сходимости к точным значениям в модели Лоренца и в модели логистического роста.

Рассмотренные в работе модели динамических систем являются нелинейными, что затрудняет использование метода наименьших квадратов для оценки их параметров. Известные авторам результаты численного эксперимента по такой оценке параметров в модели логистического роста содержат относительные ошибки порядка нескольких десятков процентов. Тогда как предложенный в работе метод оценивания параметров нелинейных динамических систем является аналитически строгим. Другое дело, если динамическая модель описывается дифференциальными уравнениями, то ее линеаризация в малой окрестности точки преобразует ее в линейную, параметры которой можно оценить методом наименьших квадратов [10]. Однако в этом случае требуется оценка получающейся погрешности при определенных значениях параметров, что является недостатком работы.

В настоящей работе, используя такие качественные свойства нелинейных рекуррентных моделей, как существование у них предельного цикла или распределения в за- висимости от значения параметров, были построены оценки параметров в трех перечисленных моделях по неточным наблюдениям. Оцениваемые параметры выражаются через средние по траектории и дисперсии ошибок наблюдений. Рассматривались аддитивная и мультипликативная модели внесения ошибок в наблюдения, при этом распределения ошибок имеют не всегда нормальный закон. Важным результатом работы является доказательство для рассмотренных моделей сходимости по вероятности построенных оценок параметров к их точным значениям, что было получено методами теории вероятностей. Проведены вычислительные эксперименты для оценки скорости этой сходимости во всех рассматриваемых моделях.

\section{1. МЕТОДЫ ИССЛЕДОВАНИЯ}

Основная идея работы состоит в представлении параметров моделей через временные средние определенных функций и оценки этих средних по наблюдениям в обход метода наименьших квадратов. Важным этапом является доказательство сходимости по вероятности оцениваемых параметров к точным значениям. Эта процедура основана на классических вероятностных неравенствах типа неравенства Чебышева.

\section{1. Средние по траектории}

Рассмотрим детерминированную рекуррентную последовательность

$$
x_{0}=a, x_{i+1}=F\left(x_{i}\right), i=0,1, \ldots,
$$

у которой $-\infty<A_{-} \leq x_{n} \leq A_{+}<\infty, n=0,1,2, \ldots$ Пусть $f(x)$ непрерывная функция на отрезке $\left[A_{-}, A_{+}\right]$, обозначим

$$
\left.\overline{f(x)}\right|_{n}=\frac{1}{n} \sum_{i=1}^{n} f\left(x_{i}\right), \overline{f(x)}=\left.\lim _{n \rightarrow \infty} \overline{f(x)}\right|_{n} .
$$

Скажем, что у последовательности $\left\{x_{i}\right\}$ существует предельный цикл $X^{1}, \ldots, X^{q}$ длины $q$, если

$$
\lim _{k \rightarrow \infty} x_{k q+j}=X^{j}, j=1, \ldots, q .
$$

Скажем, что у последовательности $\left\{x_{i}\right\}$ существует предельное распределение $p(d x)$, 
$x \in\left[A_{-}, A_{+}\right]$, где $p(d x)$ вероятностная мера на $\sigma$-алгебре измеримых по Лебегу подмножеств отрезка $\left[A_{-}, A_{+}\right]$, если для любого измеримого по Лебегу множества $C \subseteq\left[A_{-}, A_{+}\right]$справедливо равенство

$$
\lim _{n \rightarrow \infty} \frac{k(C, n)}{n}=\int_{C} p(d x)=p(C),
$$

где $k(C, n)$ число $x_{i}$, удовлетворяющих включению $x_{i} \in C, i=1, \ldots, n$.

Теорема 1. Если на отрезке $\left[A_{-}, A_{+}\right] \oint y н к-$ иия $f(x)$ возрастает, то при наличии у последовательности $\left\{x_{i}\right\}$ предельного иикла имеет место равенство

$$
\overline{f(x)}=\sum_{j=1}^{q} \frac{f\left(X^{j}\right)}{q},
$$

а при наличии предельного распределения имеет место равенство

$$
\overline{f(x)}=\int_{A_{-}}^{A_{+}} f(x) p(d x) .
$$

Доказательство. При наличии у последовательности $\left\{x_{i}\right\}$ предельного цикла утверждение очевидно. Докажем его в случае, когда у последовательности $\left\{x_{i}\right\}$ имеется предельное распределение. Возьмем произвольное $\delta>0$ и положим

$$
m=[2 / \delta]+1, \quad \gamma=\delta(2+[2 / \delta])^{-1}
$$

(здесь $[a]$ - целая часть действительного числа $a)$. Разобьем отрезок $\left[A_{-}, A_{+}\right]$на непересекающиеся подмножества

$$
\begin{gathered}
C_{j}=f^{-1}\left(\left[\frac{(j-1) \Delta}{m}+f\left(A_{-}\right), \frac{j \Delta}{m}+f\left(A_{-}\right)\right)\right), \\
\Delta=f\left(A_{+}\right)-f\left(A_{-}\right), 1 \leq j \leq m-1, \\
C_{m}=f^{-1}\left(\left[\frac{(m-1) \Delta}{m}+f\left(A_{-}\right), f\left(A_{+}\right)\right]\right) .
\end{gathered}
$$

Выберем $N(\delta)$ так, чтобы для $\forall n>N(\delta)$ выполнялось неравенство

$$
\left|\frac{k\left(C_{j}, n\right)}{n}-p\left(C_{j}\right)\right| \leq \gamma, j=1, \ldots, m .
$$

Обозначим $g(x)=\frac{f(x)-f\left(A_{-}\right)}{\Delta}$, несложно доказать для $\forall n>N(\delta)$ следующие неравенства $\frac{\sum_{x_{i} \in C_{j}, i=1, \ldots, n} g\left(x_{i}\right)}{n} \leq \frac{j}{m} \cdot \frac{k\left(C_{j}, n\right)}{n} \leq \frac{j}{m}\left(p\left(C_{j}\right)+\gamma\right) \leq$

$$
\begin{aligned}
& \leq \frac{\gamma j}{m}+\int_{C_{j}}\left(g(x)+\frac{1}{m}\right) p(d x)= \\
& =\frac{\gamma j}{m}+\frac{p\left(C_{j}\right)}{m}+\int_{C_{j}} g(x) p(d x),
\end{aligned}
$$

в свою очередь,

$$
\begin{aligned}
& \frac{\sum_{x_{i} \in C_{j}, i=1, \ldots, n} g\left(x_{i}\right)}{n} \geq \frac{j-1}{m} \cdot \frac{k\left(C_{j}, n\right)}{n} \geq \\
& \geq \frac{j-1}{m}\left(p\left(C_{j}\right)-\gamma\right) \geq \int_{C_{j}}\left(g(x)-\frac{1}{m}\right) p(d x)- \\
& -\frac{\gamma(j-1)}{m}=\int_{C_{j}} g(x) p(d x)-\frac{p\left(C_{j}\right)}{m}-\frac{\gamma(j-1)}{m} .
\end{aligned}
$$

Суммируя эти неравенства по $j=1, \ldots, m$, и используя определение $m$, получаем для $\forall n>N(\delta)$ неравенство

$$
\begin{gathered}
\overline{g(x)}-\delta \leq \overline{g(x)}-\frac{1}{m}-\frac{\gamma(m-1)}{2} \leq \\
\leq\left.\overline{g(x)}\right|_{n} \leq \frac{(m+1) \gamma}{2}+\frac{1}{m}+\overline{g(x)}=\overline{g(x)}+\delta . \\
\text { Следовательно, }\left.\overline{g(x)}\right|_{n} \rightarrow \overline{g(x)} \text { и тогда } \\
\left.\overline{f(x)}\right|_{n} \rightarrow \overline{f(x)}, n \rightarrow \infty .
\end{gathered}
$$

Теорема доказана.

Замечание. Утверждение теоремы 1 несложно распространить на случай, когда функция $f(x)$ имеет конечное число точек экстремума на отрезке $\left[A_{-}, A_{+}\right]$.

\section{2. Модель логистического роста}

Рассмотрим модель логистического роста

$$
\begin{gathered}
x_{0}=a, x_{i+1}=b x_{i}\left(1-x_{i}\right), i=0,1, \ldots, \\
0<a<1,1<b<4 .
\end{gathered}
$$

Заметим, что $0=A_{-}<x_{n}<A_{+}=1, n=0,1, \ldots$ В работе [3] доказано, что у последовательности (1) существует или предельный цикл, или предельное распределение и дана подробная классификация. Тогда последовательность $\left\{x_{i}\right\}$ удовлетворяет условиям теоремы 1 и, следовательно, у нее существуют средние по траектории $\bar{x}, x^{2}$ и выполняются соотношения

$$
0<\overline{x^{2}}<\bar{x}, b=\frac{\bar{x}}{\bar{x}-\overline{x^{2}}} .
$$

Теорема 2. Для последовательности (3) и аддитивной модели внесения очибок 
$y_{i}=x_{i}+\varepsilon_{i}$, где $\varepsilon_{i}, i=0,1, \ldots, \quad-$ n.H.o.p.c.в. $c$ $M \varepsilon_{n}=0, D \varepsilon_{n}=c$, имеет место при $n \rightarrow \infty$ сходимость по вероятности

$$
\hat{b}_{n}=\frac{\left.\bar{y}\right|_{n}}{\left.\bar{y}\right|_{n}-\left(\left.\overline{y^{2}}\right|_{n}-c\right)} \rightarrow b .
$$

Доказательство. Среднеквадратичную метрику на множестве случайных величин обозначим $\rho=\rho(x, y)=\sqrt{M(x-y)^{2}}$. Найдем пределы при $n \rightarrow \infty$ математических ожиданий и дисперсий случайных величин $\left.\bar{y}\right|_{n}$, $\left.y^{2}\right|_{n}$ :

$$
\begin{aligned}
& \left.M \bar{y}\right|_{n}=\frac{1}{n} \sum_{i=1}^{n} M\left(x_{i}+\varepsilon_{i}\right)=\left.\bar{x}\right|_{n} \rightarrow \bar{x}, \\
& \left.D \bar{y}\right|_{n}=\frac{1}{n^{2}} \sum_{i=1}^{n} D\left(x_{i}+\varepsilon_{i}\right)=\frac{1}{n^{2}} \sum_{i=1}^{n} D \varepsilon_{i}=\frac{c}{n} \rightarrow 0, \\
& \left.M \overline{y^{2}}\right|_{n}=\frac{1}{n} \sum_{i=1}^{n} M\left(x_{i}+\varepsilon_{i}\right)^{2}=\left.\overline{x^{2}}\right|_{n}+c \rightarrow \overline{x^{2}}+c \text {, } \\
& \left.D \overline{y^{2}}\right|_{n}=\frac{1}{n^{2}} \sum_{i=1}^{n} D\left(x_{i}+\varepsilon_{i}\right)^{2}=\frac{1}{n^{2}} \sum_{i=1}^{n} D\left(2 x_{i} \varepsilon_{i}+\varepsilon_{i}^{2}\right)= \\
& =\frac{1}{n^{2}} \sum_{i=1}^{n} M\left(2 x_{i} \varepsilon_{i}+\varepsilon_{i}^{2}-c\right)^{2} \leq \frac{2\left(\left.4 \tilde{n} \overline{x^{2}}\right|_{n}+d\right)}{n} \rightarrow 0 .
\end{aligned}
$$

Из полученных соотношений в силу неравенства треугольника имеем при $n \rightarrow \infty$

$$
\begin{gathered}
\rho\left(\left.\bar{y}\right|_{n}, \bar{x}\right) \leq \rho\left(\left.\bar{y}\right|_{n},\left.M \bar{y}\right|_{n}\right)+\rho\left(\left.M \bar{y}\right|_{n}, \bar{x}\right)= \\
=\sqrt{\frac{c}{n}}+|\bar{x}+\bar{x}| \rightarrow 0, \\
\leq \rho\left(\left.\overline{y^{2}}\right|_{n},\left.M \overline{y^{2}}\right|_{n}\right)+\rho\left(\left.M \overline{y^{2}}\right|_{n}, \overline{x^{2}}+c\right)= \\
=\rho\left(\left.\overline{y^{2}}\right|_{n},\left.\overline{x^{2}}\right|_{n}+c\right)+\left|\overline{x^{2}}\right|_{n}-\overline{x^{2}} \mid \leq \\
\leq \sqrt{\frac{2}{n}\left(\left.4 c \overline{x^{2}}\right|_{n}+d\right)}+\left|\overline{x^{2}}\right|_{n}-\overline{x^{2}} \mid \rightarrow 0 .
\end{gathered}
$$

Из формул (4)-(5) с помощью неравенства Чебышева получаем сходимость по вероятности

$$
\left.\bar{y}\right|_{n} \rightarrow \bar{x},\left.\overline{y^{2}}\right|_{n} \rightarrow \overline{x^{2}}+c, n \rightarrow \infty .
$$

Отсюда следует сходимость по вероятности $\hat{b}_{n} \rightarrow b, n \rightarrow \infty$. Теорема доказана.

Наряду с аддитивной моделью внесения ошибок для последовательности (1) можно рассмотреть и мультипликативную.

\section{3. Модель Рикера}

Перейдем теперь к модели Рикера

$$
\begin{gathered}
x_{i+1}=a x_{i} e^{-b x_{i}}, i=0,1, \ldots, \\
a>1, b>0, x_{0}>e^{-1} .
\end{gathered}
$$

В работах [4-7], [11] доказано, что у последовательности (4) существует или предельный цикл, или предельное распределение. Заметим, что максимум функции $a x e^{-b x}$ достигается в точке $b^{-1}$ и справедливо неравенство $x_{n} \leq \frac{a}{b e}, n=1,2, \ldots$

Логарифмируя соотношение (4), получим

$$
\ln x_{i+1}=\ln x_{i}+\ln a-b x_{i} .
$$

Если $\frac{\ln a}{b} \leq x_{i} \leq \frac{a}{b e}, \quad$ то $x_{i+1} \leq x_{i}$, а если $x_{i} \leq \frac{\ln a}{b}$, то $x_{i+1} \geq x_{i}$. Тогда в модели (4) при любых $a>1, b>0, x_{0}>0$ и $i=1,2, \ldots$ справедливы соотношения

$$
0<\min \left(x_{0}, C\right)=A_{-} \leq x_{i} \leq A_{+}=\frac{a}{b e},
$$

где $C=\min \left\{a x e^{-b x}: \frac{\ln a}{b} \leq x \leq \frac{a}{b e}\right\}$.

Из соотношения (6), утверждения теоремы 1 и замечания следует, что существуют средние по траектории $x, \overline{x^{2}}, \overline{\ln x}, \overline{x \ln x}$. Поэтому, используя равенство квадратов левой и правой части в формуле (5), нетрудно получить соотношения

$$
b=2 \frac{\overline{x \ln x}-\bar{x} \overline{\ln x}}{\bar{x}^{2}-\bar{x}^{2}}, \ln a=b \bar{x} .
$$

Введем обозначения

$$
\begin{gathered}
\hat{b}_{n}=2 e^{c / 2} \frac{\left.\overline{y \ln y}\right|_{n}-\left.c \bar{y}\right|_{n}-\left.\left.\bar{y}\right|_{n} \overline{\ln y}\right|_{n}}{\left.\overline{y^{2}}\right|_{n} e^{-c}-\left(\left.\bar{y}\right|_{n}\right)^{2}}, \\
\hat{a}_{n}=\exp \left(\left.\hat{b}_{n} \bar{y}\right|_{n} e^{-c / 2}\right) .
\end{gathered}
$$

Теорема 3. Для модели Рикера (6) и мультипликативной модели внесения ошибок $y_{i}=x_{i} \exp \left(\varepsilon_{i}\right)$, где $\varepsilon_{i}, i=0,1, \ldots,-$ n.н.o.p.c.в., имеющих нормальное распределение $c$ $M \varepsilon_{n}=0, D \varepsilon_{n}=c$, справедлива при $n \rightarrow \infty c x о-$ димость по вероятности

$$
\hat{a}_{n} \rightarrow a, \hat{b}_{n} \rightarrow b .
$$

Доказательство. Вычислим средние и дисперсии 
Оиенка параметров нелинейных рекуррентных соотномений

$$
\begin{gathered}
\left.M \bar{y}\right|_{n}=\left.\bar{x}\right|_{n} M e^{\varepsilon_{1}},\left.M \overline{y^{2}}\right|_{n}=\left.\overline{x^{2}}\right|_{n} M e^{2 \varepsilon_{1}}, \\
\left.M \overline{y \ln y}\right|_{n}=\left.\overline{x \ln x}\right|_{n} M e^{\varepsilon_{1}}+\left.\bar{x}\right|_{n} M e^{\varepsilon_{1}} \varepsilon_{1}, \\
\left.M \overline{\ln y}\right|_{n}=\left.\overline{\ln x}\right|_{n},\left.\overline{D \ln y}\right|_{n}=\frac{D \varepsilon_{1}}{n}, \\
M e^{\varepsilon_{1}} \varepsilon_{1}=c e^{c / 2}, M e^{\varepsilon_{1}}=e^{c / 2}, M e^{2 \varepsilon_{1}}=e^{2 c}, \\
\left.D \bar{y}\right|_{n}=\left.\frac{D e^{\varepsilon_{1}}}{n} \overline{x^{2}}\right|_{n},\left.D \overline{y^{2}}\right|_{n}=\left.\frac{D e^{2 \varepsilon_{1}}}{n} \overline{x^{4}}\right|_{n}, \\
\left.D \overline{y \ln y}\right|_{n}=\left.\frac{D e^{\varepsilon_{1}}}{n} \overline{(x \ln x)^{2}}\right|_{n}+\left.\frac{D e^{\varepsilon_{i}} \varepsilon_{1}}{n} \overline{x^{2}}\right|_{n}, \\
D e^{\varepsilon_{1}}=e^{2 c}-e^{c}, D e^{2 \varepsilon_{1}}=e^{8 c}-e^{4 c}, \\
D e^{\varepsilon_{1}} \varepsilon_{1}=c^{2}\left(e^{2 c}-e^{c}\right)+c e^{2 c} .
\end{gathered}
$$

Из этих соотношений следует сходимость по вероятности при $n \rightarrow \infty$

$$
\begin{aligned}
& \left.\bar{y}\right|_{n} \rightarrow \bar{x} e^{c / 2},\left.\overline{y^{2}}\right|_{n} \rightarrow \overline{x^{2}} e^{2 c},\left.\overline{\ln y}\right|_{n} \rightarrow \overline{\ln x}, \\
& \left.\overline{y \ln y}\right|_{n} \rightarrow \overline{x \ln x} e^{c / 2}+c e^{c / 2} \bar{x} \text {. }
\end{aligned}
$$

Отсюда следует сходимость по вероятности $\hat{a}_{n} \rightarrow a, \hat{b}_{n} \rightarrow b, n \rightarrow \infty$. Теорема 3 доказана.

\section{4. Модель Лоренца}

Остановимся теперь на модели Лоренца [12], представимой системой дифференциальных уравнений

$$
\left\{\begin{array}{l}
\frac{d x^{(1)}}{d t}=\sigma\left(x^{(2)}-x^{(1)}\right) \\
\frac{d x^{(2)}}{d t}=x^{(1)}\left(r-x^{(3)}\right)-x^{(2)}, \\
\frac{d x^{(3)}}{d t}=x^{(1)} x^{(2)}-b x^{(3)}
\end{array}\right.
$$

Обозначим $\quad x^{(k)}(i h)=x_{i}^{(k)}, \quad k=1,2,3$, $i=0,1, \ldots$, и выпишем для системы дифференциальных уравнений (11) систему разностных уравнений

$$
\left\{\begin{array}{l}
x_{i+1}^{(1)}=x_{i}^{(1)}+\sigma h\left(x_{i}^{(2)}-x_{i}^{(1)}\right) \\
x_{i+1}^{(2)}=x_{i}^{(2)}+\left(x_{i}^{(1)}\left(r-x_{i}^{(3)}\right)-x_{i}^{(2)}\right) h \\
x_{i+1}^{(3)}=x_{i}^{(3)}+\left(x_{i}^{(1)} x_{i}^{(2)}-b x_{i}^{(3)}\right) h .
\end{array}\right.
$$

Приведенный в $[12,13]$ анализ последовательности $\left(x_{i}^{(1)}, x_{i}^{(2)}, x_{i}^{(3)}\right), i=0,1, \ldots$, позволяет установить существование средних по траектории $\overline{x^{(k)}}, \overline{\left(x^{(k)}\right)^{2}}, \overline{x^{(k)} x^{(j)}}, k \neq j$.

Из квадрата первого равенства, а также второго и третьего равенств в формуле (8) получим

$$
\begin{gathered}
\sigma h=2 \frac{\overline{\left(x^{(1)}\right)^{2}}-\overline{x^{(1)} x^{(2)}}}{\overline{\left(x^{(1)}\right)^{2}}+\overline{\left(x^{(2)}\right)^{2}}-2 \overline{x^{(1)} x^{(2)}}}, \\
r=\frac{\overline{x^{(2)}}+\overline{x^{(1)} x^{(3)}}}{\overline{x^{(1)}}}, b=\frac{\overline{x^{(1)} x^{(2)}}}{\overline{x^{(3)}}} .
\end{gathered}
$$

Пусть наблюдения $y_{i}^{(k)}=x_{i}^{(k)}+\varepsilon_{i}^{(k)}$, $k=1,2,3, i=0,1, \ldots$, где $\varepsilon_{i}^{(k)}-$ п.н.о.р.с.в., имеющих нулевые средние и дисперсии $c^{(k)}<\infty$. Обозначим

$$
\begin{gathered}
(\hat{\sigma h})_{n}=\frac{2\left(\left.\overline{\left(y^{(1)}\right)^{2}}\right|_{n}-c^{(1)}-\left.\overline{y^{(1)} y^{(2)}}\right|_{n}\right)}{\left.\overline{\left(y^{(1)}\right)^{2}}\right|_{n}-c^{(1)}+\left.\overline{\left(y^{(2)}\right)^{2}}\right|_{n}-c^{(2)}-\left.2 \overline{y^{(1)} y^{(2)}}\right|_{n}}, \\
\hat{b}_{n}=\frac{\left.\overline{y^{(1)} y^{(2)}}\right|_{n}}{\left.\overline{y^{(3)}}\right|_{n}}, \hat{r}_{n}=\frac{\left.\overline{y^{(2)}}\right|_{n}-c^{(2)}+\left.\overline{y^{(1)} y^{(3)}}\right|_{n}}{\left.\overline{y^{(1)}}\right|_{n}} .
\end{gathered}
$$

Теорема 4. Справедлива при $n \rightarrow \infty$ сходимость по вероятности

$$
(\hat{\sigma h}) \rightarrow \sigma h, \hat{b}_{n} \rightarrow b, \hat{r}_{n} \rightarrow r
$$

Доказательство. По аналогии с доказа-тельством теоремы 2 нетрудно установить сходимость по вероятности при $n \rightarrow \infty$

$$
\begin{gathered}
\left.\overline{y^{(k)}}\right|_{n} \rightarrow \overline{x^{(k)}}, k=1,2,3, \\
\left.\overline{\left(y^{(j)}\right)^{2}}\right|_{n} \rightarrow \overline{\left(x^{(j)}\right)^{2}}+c^{(j)}, j=1,2 .
\end{gathered}
$$

Докажем теперь сходимость по вероятности при $n \rightarrow \infty$

$$
\left.\left.\overline{y^{(1)} y^{(2)}}\right|_{n} \rightarrow \overline{x^{(1)} x^{(2)}}\right|_{n},\left.\left.\overline{y^{(1)} y^{(3)}}\right|_{n} \rightarrow \overline{x^{(1)} x^{(3)}}\right|_{n} .
$$

Остановимся на первом соотношении, т.к. второе доказывается аналогично, и для этого вычислим среднее и дисперсию

$$
\left.M \overline{y^{(1)} y^{(2)}}\right|_{n}=\left.\overline{x^{(1)} x^{(2)}}\right|_{n},
$$

$$
\left.\overline{D y^{(1)} y^{(2)}}\right|_{n}=\frac{1}{n}\left[\left.c^{(2)} \overline{\left(x^{(1)}\right)^{2}}\right|_{n}+\left.c^{(1)} \overline{\left(x^{(2)}\right)^{2}}\right|_{n}\right] \text {. }
$$

Из полученных соотношений и из формул (10) следует справедливость утверждения теоремы 4. Теорема доказана.

\section{2. РЕЗУЛЬТАТЫ И ИХ ОБСУЖДЕНИЕ}

В предыдущем разделе для модели логистического роста, модели Рикера и дискретизированной модели Лоренца доказаны сходимости по вероятности построенных оценок параметров к их точным значениям. В этом разделе проведены вычислительные эксперименты для оценки скорости этой сходимости. 
Оценки всех параметров были вычислены 100 раз и построены гистограммы частот полученных значений с 5 интервалами.

\section{Модель логистического роста}

Расчеты велись для случая $a=0,5, b=3$ при $n=1000$. Рассматривалась аддитивная модель внесения ошибок в предположении, что $\varepsilon_{i}, i=1, . ., n$, имеют равномерное распределение на отрезке $[-1 / 4,1 / 4]$. Результаты вычислений приведены в табл. 1 и на рис. 1.

Таблица 1. Интервальное распределение $\hat{b}_{n}$ [Table 1. Interval distribution $\hat{b}_{n}$ ]

\begin{tabular}{|c|c|}
\hline интервалы изменения $\hat{b}_{n}$ & частота интервала \\
\hline $2.89963-2.94201$ & 7 \\
\hline $2.94201-2.98438$ & 25 \\
\hline $2.98438-3.02676$ & 43 \\
\hline $3.02676-3.06914$ & 21 \\
\hline $3.06914-3.11151$ & 3 \\
\hline
\end{tabular}

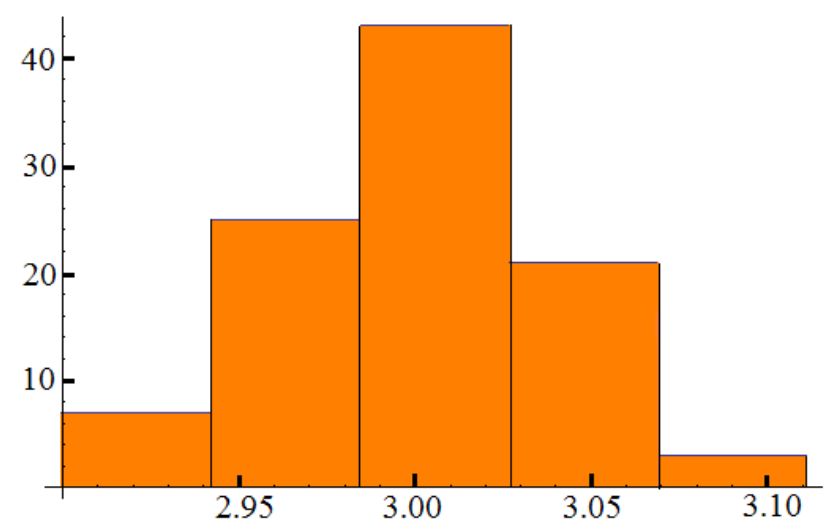

Puc. 1. Гистограмма частот для $\hat{b}_{n}$

[Fig 1. Frequency histogram for $\hat{b}_{n}$ ]

\section{Модель Рикера}

Расчеты велись для случая $x_{0}=1,01$, $a=300, b=1$ при $n=1000$. Рассматривалась мультипликативная модель внесения ошибок в предположении, что $\varepsilon_{i}, i=1, . ., n$, имеют нормальный закон рспеределения со средним ноль и дисперсией $c=1 / 48$. Результаты вычислений в модели Рикера приведены в табл. 2 и на рис. 2.
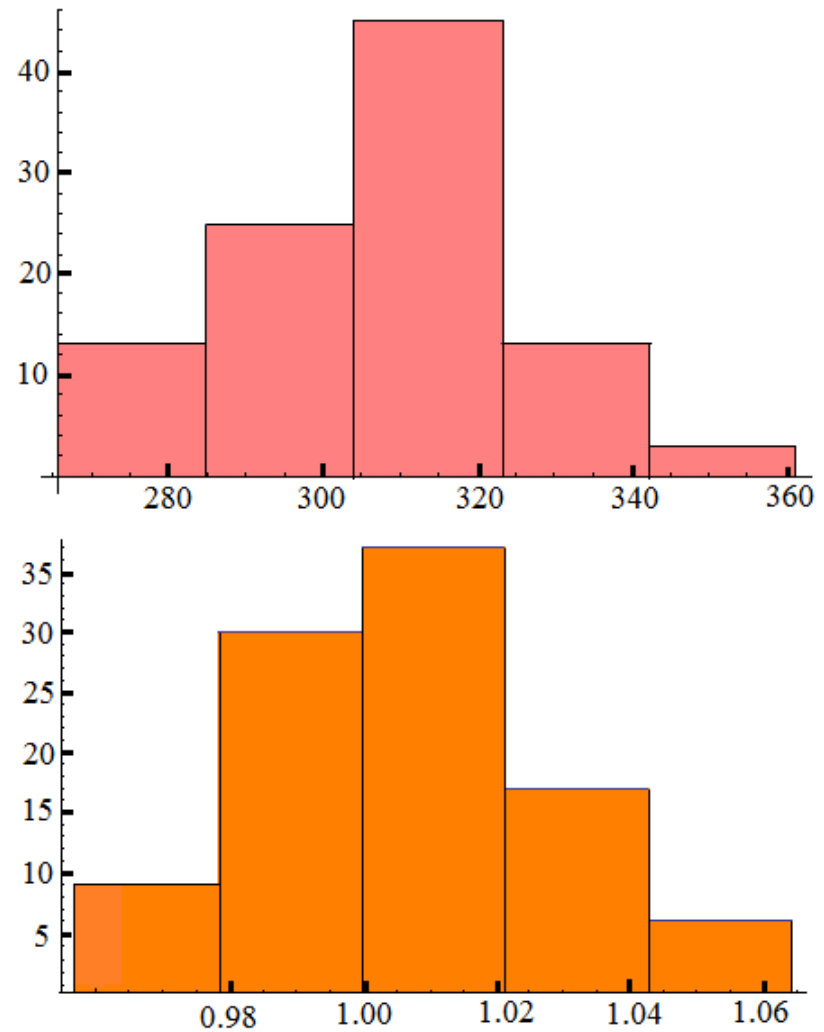

Рис. 2. Гистограмми частот для $\hat{a}_{n}$ (верхняя) и $\hat{b}_{n}$ (нижняя)

[Fig. 2. Frequency histograms for $\hat{a}_{n}$ (upper) and $\hat{b}_{n}$ (lower)]

Таблица 2. Интервальные распределения $\hat{a}_{n}, \hat{b}_{n}$ [Table 2. Interval distributions $\hat{a}_{n}, \hat{b}_{n}$ ]

\begin{tabular}{|c|c|}
\hline интервалы изменения $\hat{a}_{n}$ & частота интервала \\
\hline $265.562-284.761$ & 13 \\
\hline $284.761-303.96$ & 25 \\
\hline $303.96-323.159$ & 45 \\
\hline $323.159-342.358$ & 13 \\
\hline $342.358-361.556$ & 3 \\
\hline интервалы изменения $\hat{b}_{n}$ & частота интервала \\
\hline $0.956914-0.9784$ & 9 \\
\hline $0.9784-0.999885$ & 30 \\
\hline $0.999885-1.02137$ & 37 \\
\hline $1.02137-1.04286$ & 17 \\
\hline $1.04286-1.06434$ & 6 \\
\hline
\end{tabular}

\section{Модель Лоренца}

Расчеты велись для случая $x_{0}^{(1)}=1, x_{0}^{(2)}=2$, $x_{0}^{(3)}=3, h=0,1, \sigma=19, b=3, r=2, n=200$. 
Рассматривалась аддитивная модель внесения ошибок, полагая, что $\varepsilon_{i}, i=1, . ., n$, имеют нормальный закон со средним ноль и дисперсией $c=1 / 48$. Результаты приведены в табл. 3 и на рис. 3.

Таблица 3. Интервальные распределения

$$
(\sigma h)_{n}, \hat{b}_{n}, \hat{r}_{n}
$$

[Table 3. Interval distributions $(\hat{\sigma} h)_{n}, \hat{b}_{n}, \hat{r}_{n}$ ]

\begin{tabular}{|c|c|}
\hline $\begin{array}{c}\text { интервалы изменения } \\
(\hat{\sigma h})_{n}\end{array}$ & частота интервала \\
\hline $1.85497-1.87214$ & 11 \\
\hline $1.87214-1.88931$ & 22 \\
\hline $1.88931-1.90648$ & 43 \\
\hline $1.90648-.92366$ & 18 \\
\hline $1.92366-1.94083$ & 5 \\
\hline интервалы изменения $\hat{b}_{n}$ & частота интервала \\
\hline $2.85799-2.89735$ & 11 \\
\hline $2.89735-2.93672$ & 23 \\
\hline $2.93672-2.97608$ & 36 \\
\hline $2.97608-3.01544$ & 26 \\
\hline $3.01544-3.05481$ & 3 \\
\hline интервалы изменения $\hat{r}_{n}$ & частота интервала \\
\hline $1.95884-1.97568$ & 14 \\
\hline $1.97568-1.99251$ & 26 \\
\hline $1.99251-2.00935$ & 40 \\
\hline $2.00935-2.02619$ & 17 \\
\hline $2.02619-2.04303$ & 2 \\
\hline
\end{tabular}

\section{ЗАКЛЮЧЕНИЕ}

Результаты вычислительного эксперимента показали достаточно хорошую близость оценок параметров в моделях логистического роста, Рикера и дискретизированной модели Лоренца к оцениваемым параметрам. Следует отметить, что приближение средней по траектории $\overline{f(x)}$ детерминированной функции $f(x)$ с помощью среднего по конечному отрезку $\left.\overline{f(x)}\right|_{n}$ исследуется в теории динамических систем. Тогда как близость среднего $\left.\overline{f(y)}\right|_{n}$, вычисленного по неточным наблюдениям с соответствующими добавками к среднему $\left.\overline{f(x)}\right|_{n}$ оценивается вероятностными методами. В последнее время появилось большое
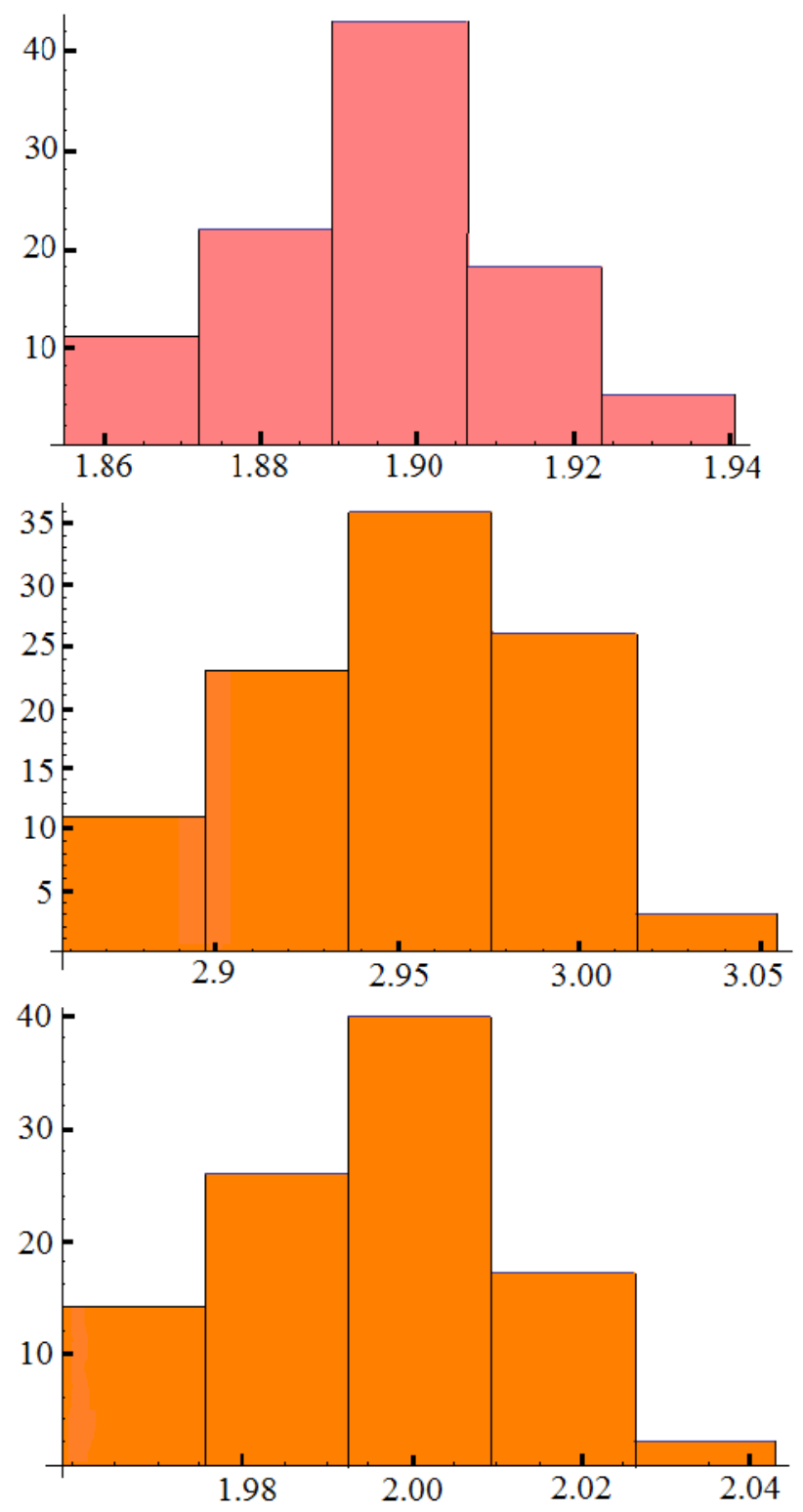

Рис. 3. Гистограммы частот для $(\hat{\sigma h})_{n}$ (верхняя), $\hat{b}_{n}$ (в середине), $\hat{r}_{n}$ (нижняя)

[Fig. 3. Frequency histograms for $(\hat{\sigma} h)_{n}$ (top),

$$
\hat{b}_{n} \text { (middle), } \hat{r}_{n} \text { (bottom)] }
$$

количество работ по моделированию популяционной динамики на основе нелинейных рекуррентных соотношений [14-18]. Предлагаемые в статье методы могут быть применены к оценкам параметров этих моделей.

\section{КОНФЛИКТ ИНТЕРЕСОВ}

Авторы декларируют отсутствие явных и потенциальных конфликтов интересов, связанных с публикацией настоящей статьи. 


\section{Г. Ш. Цициашвили, М. А. Осипова}

\section{СПИСОК ЛИТЕРАТУРЫ}

1. Dynamics of a discrete-time stage-structured predator-prey system with Holling type II response function / G.P. Neverova [and other] // Nonlinear Dynamics. 2019. - V. 98. - P. 427-446. Doi: 10.1007/s11071-019-05202-3.

2. Neverova, G. P. Changes in population dynamics regimes as a result of both multistability and climatic fluctuation / G. P. Neverova, M. P. Kulakov, E. Ya. Frisman // Nonlinear Dynamics. - 2019. - V. 97. - P. 107-122. Doi: 10.1007/s11071-019-04957-z.

3. Шарковский, А. Н. Разностные уравнения и динамика численности популяций / А. Н. Шарковский. - Препринт 82.18. - Киев : Институт математики АН УССР, 1982. - 22 с.

4. Complex patterns in a space-and time discrete predator-prey model with Beddington-DeAngelis functional response / T. Huang [and other] // Communications in Nonlinear Science and Numerical Simulation. - 2017. - V. 43. P. 182-199. Doi: 10.1016/j.cnsns.2016.07.004.

5. Шапиро, А. П. Реккурентные уравнения в теории популяционной биологии / А. П. Шапиро, С. П. Луппов. - Москва : Наука, 1983. - $136 \mathrm{c}$.

6. Пых, Ю. А. Равновесие и устойчивость в моделях популяционной динамики / Ю. А. Пых. - Москва : Наука, 1983. - 184 с.

7. Kundu, S. Dinamics of a delayed predatorprey system with stage structure and cooperation for preys / S. Kundu, S. Maitra // Chaos, Solitons \& Fractals. - 2018. - V. 114. - P. 453-460. Doi: 10.1016/j.chaos.2018.07.013

8. Талалаева, А. Б. Оценка параметров модели Рикера по неточным наблюдениям/ А. Б. Талалаева, Г. Ш. Цициашвили // Сибирский экологический журнал. - 1999. - Т. 4. С. 377-379.

9. Герасин, С. Н. Оценка параметров численности популяции в дискретной модели Рикера / С. Н. Герасин, М. А. Козлов // Бионика интеллекта. - 2008. - № 2 (69). - С. 159-163.

10. Буляница, А. Л. Методы оценивания параметров кривой логистического роста. Ч. 1. Оптимизация условий оценивания при наличии аддитивной случайной помехи / А. Л. Буляница // Научное приборостроение. - 2009. - Т. 19, № 3. - С. 3-11.
11. Huang, T. Bifurcation, chaos and pattern formation in a space-and time discrete predator-prey system / T. Huang, H. Zhang // Chaos, Solitons \& Fractals. - 2016. - V. 91. - P. 92-107. Doi: 10.1016/j.chaos.2016.05.009.

12. The Lyapunov dimension formula for the global attractor of the Lorenz system/ G. A. Leonov [and other] // Communications in Nonlinear Science and Numerical Simulation.2016. - V. 41. - P. 84-103. Doi: 10.1016/j.cnsns.2016.04.032.

13. Lorenz, E. N. Deterministic non-periodic flow / E. N. Lorenz // Journal of the Atmospheric Sciences. - 1963. - V. 20. - P. 131-141.

14. Density feedbacks mediate effects of environmental change on population dynamics of a semidesert rodent / C.R. Nater [and other] // Journal of Animal Ecology. - 2018. - V. 87, No 6. P. 1534-1546. Doi: 10.1111/1365-2656.12888.

15. Neverova, G. P. Dinamic models of population size and its genetic structure for species with nonoverlapping generations and stage development / G. P. Neverova, E. Ya. Frisman // Communications in Nonlinear Science and Numerical Simulation. - 2021. - V. 94. - No 105554. Doi: 10.1016/j.cnsns.2020.105554.

16. Математическое моделирование популяционной динамики на основе рекуррентных уравнений: результаты и перспективы. Ч. I / Е. Я. Фрисман // Известия РАН. Серия биологическая. - 2021. - № 1. - С. 3-18. Doi: $10.31857 / \mathrm{s} 0002332921010069$.

17. Фрисман, Е. Я. Модельные динамические популяционные режимы в сообществах с циклирующими видами: синхронизация, параметрический резонанс и мультистабильность на примере популяций, динамика которых описывается моделью Рикера / Е. А. Фрисман, К. В. Шлюфман, Г. П. Неверова // Известия РАН. Серия биологическая. - 2019. - № 4. - С. 341-352. Doi: 10.1134/ s0002332919040052.

18. Шлюбман, К. В. Динамические режимы модели Рикера с периодически изменяющимся мальтузианским параметром / К. В. Шлюфман, Г. П. Неверова, Е. Я. Фрисман // Нелинейная динамика. - 2017. - Т. 13, № 3. C. 363-380. Doi: 10.20537/nd1703005. 
Цициашвили Гурами Шалвович - д-р физ.-мат. наук, профессор, главный научный сотрудник Института прикладной математики ДВО РАН.

E-mail: guram@iam.dvo.ru

ORCID iD: https://orcid.org/0000-0003-2600-0474

Осипова Марина Анатольевна - канд. физ.-мат. наук, доцент кафедры алгебры, геометрии и анализа Дальневосточного федерального университета, научный сотрудник Института прикладной математики ДВО РАН.

E-mail:mao1975@list.ru

ORCID iD: https://orcid.org/0000-0001-5615-9449

DOI: https://doi.org/10.17308/sait.2021.3/3733

Received 26.08.2021

Accepted 20.11.2021

ISSN 1995-5499

\title{
ESTIMATION OF PARAMETERS OF NONLINEAR RECURRENT RELATIONS
}

\author{
(C) 2021 G. Sh. Tsitsiashvili, M. A. Osipova \\ Institute of Applied Mathematics FEB RAS \\ 7, Radio Street, 690041 Vladivostok, Russian Federation \\ Far Eastern Federal University \\ 10, Ajax, Russian Island, 690922 Vladivostok, Russian Federation
}

\begin{abstract}
Annotation. Abstract. In this paper, estimates of the parameters of nonlinear recurrent sequences are constructed from inaccurate observations. We are talking about the logistic growth model, the Rikker model and the discretized Lorentz model. In the Lorentz model, the differential problem was reduced to a finite-difference scheme. Additive and multiplicative models of introducing errors into observations are considered, while the error distributions do not always have a normal distribution law. The main idea of the work is to represent the parameters of models through the time averages of certain functions and to estimate these averages from observations. The questions of the existence of time averages of some functions of model variables are the subject of the theory of dynamical systems. They are determined by the presence of limit cycles or limit distributions of a dynamical system. Since dynamic systems are observed against the background of random errors, the estimated parameters are expressed in terms of the trajectory averages and in terms of the variance of the observation errors. An important step in this work was the proof of the convergence in probability of the estimated parameters of a deterministic system to exact values. This procedure is based on classical probabilistic inequalities of the Chebyshev inequality type. The obtained results are verified in the course of computational experiments, in which polygons of the frequencies of the estimated parameters are constructed and compared with their exact values. The models of dynamical systems considered in this paper are nonlinear, which makes it difficult to use the least squares method to estimate their parameters. The results of a numerical experiment known to the authors for such an estimation of parameters contain rather large errors. Whereas the method proposed in this paper for estimating the parameters of nonlinear dynamical systems is analytically rigorous.
\end{abstract}

Keywords: nonlinear recurrent sequences, parameter estimates, probability convergence, limit cycles and distributions.

Osipova Marina A.

e-mail: mao1975@list.ru 


\section{CONFLICT OF INTEREST}

The authors declare the absence of obvious and potential conflicts of interest related to the publication of this article.

\section{REFERENCES}

1. Neverova G.P., Zhdanova O.L., Frisman E. Ya., Ghosh B. (2019) Dynamics of a discrete-time stage-structured predator-prey system with Holling type II response function // Nonlinear Dy-namics. 98. P. 427-446. DOI: 10.1007/ s11071-019-05202-3.

2. Neverova G. P., Kulakov M. P., Frisman E. Ya. (2019) Changes in population dynamics regimes as a result of both multistability and climatic fluctuation // Nonlinear Dynamics. 97. P. 107-122. DOI: $10.1007 / \mathrm{s} 11071-019-04957-z$.

3. Sharkovskij A. N. (1982) Raznostnye uravneniya i dinamika chislennosti populyacij [Dif-ferential equations and population dynamics]. Preprint 82.18. Kiev, Institut matematiki AN USSR. (in Russian)

4. Huang T., Zhang H., Yang H., Wang N., Zhang F. (2017) Complex patterns in a space-and time discrete predator-prey model with Beddington-DeAngelis functional response // Communications in Nonlinear Science and Numerical Simulation. 43. P. 182-199. DOI: $10.1016 /$ j.cnsns.2016.07.004.

5. Shapiro A. P., Luppov S. P. (1983) Rekkurentnye uravneniya $\mathrm{V}$ teorii populyacionnoj biologii [Recompetitive equations in the theory of population biology]. Moskva, Nauka. (in Russian)

6. Pyh Yu. A. (1983) Ravnovesie i ustojchivost' v modelyah populyacionnoj dinamiki [Equi-librium and stability in models of population dynamics]. Moskva, Nauka. (in Russian)

7. Kundu S., Maitra S. (2018) Dinamics of a delayed predator-prey system with stage structure and cooperation for preys // Chaos, Solitons \& Fractals. 114. P. 453-460. DOI: 10.1016/j.chaos.2018.07.013

8. Talalaeva A. B., Ciciashvili G. Sh. (1999) Ocenka parametrov modeli Rikera po netochnym nablyudeniyam [Estimation of the parameters of the Riker model based on inaccurate ob- servations] // Sibirskij ekologicheskij zhurnal. 4. P. 377-379. (in Russian)

9. Gerasin S. N., Kozlov M. A. (2008) Ocenka parametrov chislennosti populyacii $\mathrm{v}$ diskretnoj modeli Rikera [Estimation of population size parameters in a discrete Riker model] // Bionika intellekta. 2 (69)., P. 159-163. (in Russian)

10. Bulyanica A. L. (2009) Metody ocenivaniya parametrov krivoj logisticheskogo rosta. $\mathrm{CH}$. 1. Optimizaciya uslovij ocenivaniya pri nalichii additivnoj sluchajnoj pomekhi // Nauchnoe pribo-rostroenie. 19 (3). P. 3-11. (in Russian)

11. Huang T., Zhang H. (2016) Bifurcation, chaos and pattern formation in a space-and time discrete predator-prey system // Chaos, Solitons \& Fractals. 91. P. 92-107. DOI: 10.1016/j.chaos.2016.05.009.

12. Leonov G., Kuznetsov N., Korzhemanova N. A., Kusakin D. V. (2016) The Lyapunov dimension formula for the global attractor of the Lorenz system // Communications in Nonlinear Science and Numerical Simulation. 41. P. 84-103. DOI: 10.1016/j.cnsns.2016.04.032.

13. Lorenz E. N. (1963) Deterministic non-periodic flow. Journal of the Atmospheric Sciences. 20. P. 131-141.

14. Nater C. R., Benthem K. J., Canale C. I., Schradin C., Ozgul A. (2018) Density feedbacks mediate effects of environmental change on population dynamics of a semidesert // Journal of Animal Ecology. 87 (6). P. 1534-1546. DOI: 10.1111/1365-2656.12888.

15. Neverova G. P., Frisman E. Ya. (2021) Dinamic models of population size and its genetic structure for species with nonoverlapping generations and stage development // Communications in Nonlinear Science and Numerical Simulation. 94. No 105554. DOI: 10.1016/j.cnsns.2020.105554.

16. Frisman E. Ya., Zhdanova O. L., Kulakov M. P., Neverova G. P., Revuckaya O. L. (2021) Matematicheskoe modelirovanie populyacionnoj dinamiki na osnove rekurrentnyh uravnenij: rezu-l'taty i perspektivy. Ch. I. [Mathematical modeling of population dynamics based on recurrent equa-tions: results and prospects. Ch. I.] // Izvestiya RAN. Seriya biologicheskaya. 1. P. 3-18. DOI: 10.31857/s0002332921010069. (in Russian) 


\section{Оиенка параметров нелинейных рекуррентных соотномений}

17. Frisman E. Ya., Shlyufman K. V., Neverova G. P. (2019) Model'nye dinamicheskie pop-ulyacionnye rezhimy $\mathrm{v}$ soobshchestvah s cikliruyushchimi vidami: sinhronizaciya, parametricheskij rezonans i mul'tistabil'nost' na primere populyacij, dinamika kotoryh opisyvaetsya model'yu Rikera [Model dynamic population regimes in communities with cycling species: synchronization, para-metric resonance and multistability on the example of populations whose dynamics is described by the Riker model] // Izvestiya
RAN. Seriya biologicheskaya. 4. P. 341-352. DOI: 10.1134/s0002332919040052. (in Russian)

18. Shlyufman K. V., Neverova G. P., Frisman E. Ya. (2017) Dinamicheskie rezhimy modeli Rikera s periodicheski izmenyayushchimsya mal'tuzianskim parametrom [Dynamic modes of the Riker model with a periodically changing Malthusian parameter] // Nelinejnaya dinamika. 13 (3). P. 363-380. DOI: 10.20537/nd1703005. (in Russian)

Tsitsiashvili Gurami Sh. - DSc in Physics and Mathematics, Professor, Main Scientific Researcher, Institute for Applied Mathematics FEB RAS.

E-mail: guram@iam.dvo.ru

ORCID iD: https://orcid.org/0000-0003-2600-0474

Osipova Marina A. - PhD in Physics and Mathematics, Associate Professor, Far Eastern Federal University, Main Scientific Researcher, Institute for Applied Mathematics FEB RAS.

E-mail: mao1975@list.ru

ORCID iD: https://orcid.org/0000-0001-5615-9449 